\section{The relationship between cellulose nanocrystal dispersion and strength}

$$
\text { Yizheng Cao }{ }^{1} \text {, Pablo Zavattieri }{ }^{2} \text {, Jeffrey Youngblood }{ }^{1} \text {, Robert } \text { Moon }^{3} \text {, and Jason Weiss }{ }^{4}
$$

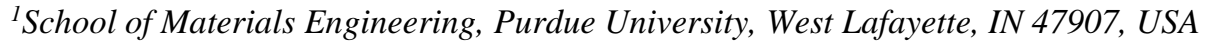

${ }^{2}$ School of Civil Engineering, Purdue University, West Lafayette, IN 47907, USA

${ }^{3}$ Forest Products Laboratory, US Forest Service, Madison, WI 53726, USA

${ }^{4}$ School of Civil and Construction Engineering, Oregon State University, OR 97331, USA

\title{
Abstract
}

This paper studies the agglomeration of cellulose nanocrystals (CNCs) and uses ultrasonication to disperse CNCs in cement pastes in an attempt to improve strength. Rheological measurements show that when the concentration of CNCs exceeds $1.35 \%$ by volume in deionized water, agglomerates start to develop. This experimental finding is comparable to the value obtained from a geometrical percolation model ( $1.38 \%$ by volume). When the matrix phase (deionized water) is replaced with a simulated cement paste pore solution, the CNCs begin to agglomerate at a lower concentration (approximately $0.18 \%$ by volume). The $\mathrm{CNC}$ concentration of $0.18 \%$ corresponds to the concentration of CNCs in cement paste where the maximum strength is reached. Tip ultrasonication was found to effectively disperse the CNCs and the cement pastes obtained strength improvements of up to 50\%, which is significantly better than the strength improvement of raw CNCs alone (20 30\%).

Key words: cellulose nanocrystal, agglomeration, ultrasonication, flexural strength, short circuit diffusion

\section{Introduction}

Cellulose nanocrystals (CNCs) are promising materials that can be used for nanoscale reinforcing [1]. They are typically $0.05-0.5 \mu \mathrm{m}$ in length and 3-5 $\mathrm{nm}$ in width. CNCs have a number of unique benefits, such as a high aspect ratio, high elastic modulus, high strength, low density, and reactive surfaces that are able to be functionalized [1, 2]. Compared with other typical nano-reinforcing materials, CNCs are environmentally friendly because they are renewable, biodegradable, sustainable, have low toxicity and low cost (estimated production costs of $<\$ 10 / 1 b$ ), and the extraction processes has low environmental and safety risks $[1,3,4]$. 
Cement pastes that contain CNCs have shown an improvement in flexural strength of $20 \%$ to $30 \%$ [5]. The increase in the strength appears to be related to an increase in the degree of hydration (DOH) of the cement pastes. Two mechanisms are used to explain the increase in DOH by CNCs: steric stabilization and short circuit diffusion (SCD) [5]. Steric stabilization is the mechanism that some water reducing agents (WRAs) use to increase the workability in fresh cement suspensions. SCD is a mechanism in which the CNCs adsorbed on the cement particles surface and transport water from the capillary pores to the unhydrated cement. The strength improvement reaches a maximum value at a $\mathrm{CNC}$ concentration of $0.2 \%$ by volume. At higher $\mathrm{CNC}$ concentrations a decrease in strength is observed which is believed to be caused by CNC agglomeration. The potential for agglomeration is supported with zeta potential and rheological measurements [5]. It is hypothesized that if the CNC agglomeration can be minimized, further improvements in the strength of CNC-cements composites can be achieved. When a high concentration of CNCs are added into the matrix, they tend to form agglomerates which are likely to act as defects or stress concentrators (Fig. 1). With a better dispersion processing, the CNCs may be able to be more uniformly distributed in the matrix. As such, there are two objectives for this paper: (1) to understand how the CNC agglomerates are formed in the cement matrix and how they are related with the mechanical performance of cement pastes; (2) to evaluate methods to disperse CNCs in order to further improve the mechanical performance. Towards these ends, the following specific questions need to be answered: What are percolation thresholds of CNCs in different matrices (e.g., cement pore solution) and are they related with the rheological and mechanical properties of cement paste? Can sonication or chemical admixtures disperse CNCs in aqueous suspensions, and are they still dispersed after mixing with cement? Can CNCs dispersion lead to an improvement in mechanical performance of cement paste?
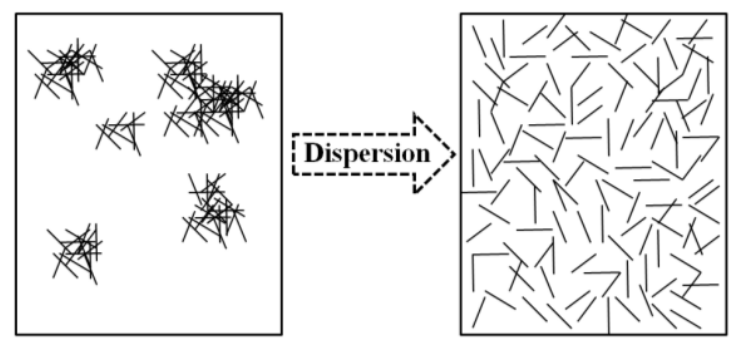

Fig. 1. A schematic of the CNC agglomerates and how they are removed with a dispersion processing 
In this paper, the agglomeration of CNCs is studied with a fundamental analysis by percolation theory, in which the percolation threshold is calculated or measured for $\mathrm{CNCs}$ within different matrices. Percolation theory has been developed by Broadbent and Hammersley [6] and has been applied in problems such as fiber-polymer nanocomposites [7], resin with carbon black [8], sol-gel materials [9] and cement based materials [10-12]. While percolation originated from a mathematical concept [13], it can be understood as the formation of a threedimensional continuous network of connecting particles through the matrix phase [8, 14]. The minimum volume fraction or concentration of the particles needed to reach percolation is referred as the percolation threshold or critical concentration $[8,13,15]$. When the percolation threshold is reached, the bulk material properties are subject to abrupt changes, such as thermal conductivity [16], storage modulus [7], and dielectric constant [17]. These changes are associated with the transitions, e.g., from insulator to conductor in the case of electrical conductivity [7, 18]. The role of the particle agglomeration has been reported to be strongly related to the percolation for different materials systems, such as carbon black-epoxy [8], carbon nanotube-epoxy composites [14], and thin film metal/silicon [19]. Sieradzki et al. [19] found that for the system $\mathrm{Ag} / \mathrm{SiO} \mathrm{O}_{2} / \mathrm{Si}(100)$, the agglomeration facilitates percolation. Schueler et al. $[8,20]$ explained the percolation-like transition in electrical conductivity by the agglomeration of carbon black. They also reported that in the carbon black-resin composites, larger individual agglomerates led to a lower percolation threshold. Further they reported that percolation cannot be reached without the formation of agglomerates. Alig et al. [21] observed agglomeration and found that the insulator-conductor transition is a result of interconnected carbon nanotube agglomerates. Pegel et al. [22] reported that the agglomeration enhanced the development of the percolated network in the carbon nanotube-polycarbonate composites. Martin et al. [23] used high temperature to promote the formation of the carbon nanotube agglomerates in order to reach a low percolation threshold. The percolation threshold of CNCs is studied in this work using rheological measurements in an attempt to correlate agglomeration with flexural strength of cement pastes.

The dispersion of CNCs can potentially improve the mechanical properties by removing agglomerates which act as defects or stress concentrators. There have been two common methods to disperse the agglomerated nanoparticles in the literature. The first method is to use mechanical energy to separate the particles. The second method alter is to the surface energy of the particles by surfactants [24]. The mechanical approach has used high shear mixing and/or ultrasonication (abbreviated as sonication) to break agglomerates [24-26]. The second method improves chemical compatibility between the particles and the matrix phase to disperse the agglomerates. In this study, both methods 
79 are used. First, a tip-sonication is performed to disperse the CNCs in water. Second, one typical WRA is used to 80 help the dispersion of CNCs. For some other nano-scale fiber materials, such as carbon nanotubes, Vaisman et al.

81 [24] and Konsta-Gdoutos et al. [25, 26] have demonstrated that WRA can help the dispersion in conjunction with 82 sonication.

83

84 2. Materials and specimen preparation

85 A Type V cement was used in this investigation due to its low aluminate and ferrite phase content. The Bogue composition and Blaine fineness of the Type V cement are shown in Table 1.

Table 1. Bogue compositions of Type V cement

88

\begin{tabular}{c|c}
\hline $\mathrm{C}_{3} \mathrm{~S}(\%)$ & 63.8 \\
\hline $\mathrm{C}_{2} \mathrm{~S}(\%)$ & 13.0 \\
\hline $\mathrm{C}_{3} \mathrm{~A}(\%)$ & 0 \\
\hline $\mathrm{C}_{4} \mathrm{AF}(\%)$ & - \\
\hline $\mathrm{C}_{4} \mathrm{AF}+\mathrm{C}_{2} \mathrm{~F}(\%)$ & 12.6 \\
\hline Blaine fineness, $\mathrm{m}^{2} / \mathrm{kg}$ & 316 \\
\hline
\end{tabular}

Two different CNC materials were used in this work, both of which are manufactured by the USDA Forest Service-Forest Products Laboratory. The CNCs were extracted from Eucalyptus dry-lap cellulose fibers using sulfuric acid hydrolysis. The CNCs have a $0.81 \mathrm{wt}$ \% surface-grafted sulfate content. The first CNC material is in a form of suspension (5.38 wt. \% CNCs in water), while the second one is in the form of a freeze dried powder.

\subsection{Cement paste preparation}

For all the cement pastes, the CNCs were always introduced into the mixing process after the cement. This step was followed by the addition of extra water. The water to cement ratio (w/c) was kept at a constant value of 0.35 . The mixing procedures are described in a previous work [5]. The mixtures proportions are listed in Table 2. The critical CNC concentration or percolation threshold is based on the geometry and distribution of the CNCs, as such Table 2 lists both the mass and the volume fractions. The 7 mixtures are classified with the volume ratio between CNC and cement (the second last column), from $0 \%$ to $1.5 \%$. 
To disperse the CNCs, sonication was performed with a Hielscher Sonic Processor UP200S with a half-inch tip.

The mechanical vibrations from the tip are transferred into the suspension, resulting in continuous formation and collapse of microscopic bubbles, and the energy release by the bubbles enables the dispersion of the CNC agglomerates [25-27]. During sonication, the suspension container is placed in an ice bath to avoid the subsequent temperature rise of the suspension as a result of the sonic waves. The container was a cylinder with a diameter of 6 $\mathrm{cm}$, which was intended to produce a uniform dispersion in the radial direction. The container was also designed with a small mouth with a diameter close to the diameter of the tip in order to reduce water evaporation during sonication. After sonication, the suspensions are kept at ambient for at least 3 hours prior to mixing with cement to minimize the influence of the low temperature due to the ice bath.

Table 2. Experimental matrix for cement pastes with CNCs

\begin{tabular}{|c|c|c|c|c|c|c|c|c|}
\hline \multirow{2}{*}{ Mixture } & \multicolumn{3}{|c|}{ mass (g) } & \multicolumn{3}{c|}{ volume $\left(\mathrm{cm}^{3}\right)$} & CNC/cement & CNC/(CNC+water) \\
\cline { 2 - 6 } & cement & water & CNC & cement & water & CNC & vol. \% & vol. \% \\
\hline 1 (ref) & 500 & 175 & 0 & 160.3 & 175 & 0 & $0.00 \%$ & $0.00 \%$ \\
2 & 500 & 175 & 0.103 & 160.3 & 175 & 0.064 & $0.04 \%$ & $0.04 \%$ \\
3 & 500 & 175 & 0.256 & 160.3 & 175 & 0.160 & $0.10 \%$ & $0.09 \%$ \\
4 & 500 & 175 & 0.513 & 160.3 & 175 & 0.321 & $0.20 \%$ & $0.18 \%$ \\
5 & 500 & 175 & 1.282 & 160.3 & 175 & 0.801 & $0.50 \%$ & $0.46 \%$ \\
6 & 500 & 175 & 2.564 & 160.3 & 175 & 1.603 & $1.00 \%$ & $0.91 \%$ \\
7 & 500 & 175 & 3.846 & 160.3 & 175 & 2.404 & $1.50 \%$ & $1.36 \%$ \\
\hline
\end{tabular}

113

114 3. Experimental Methods

115 3.1. Rheological experiments

116 Rheological experiments were performed to quantify the dispersion and agglomeration of CNCs in deionized (DI)

117 water and simulated pore solution. These techniques have been described in a previous work [5]. To evaluate the 118 influence of ionic concentration on the rheological properties of CNCs in water, two charged systems were used. 119 The first system was a calcium nitrate $\left(\mathrm{Ca}\left(\mathrm{NO}_{3}\right)_{2}\right)$ solution and the second system a simulated pore solution. These 
two systems were compared with DI water. The simulated pore solution was prepared based on the 1 hour composition reported by Rajabipour et al. [28] as shown in Table 3. However the solution prepared according to

122 Table 3 has a higher $\mathrm{pH}$ than the fresh Type V cement paste used in this work. The solution was then diluted (4:1)

123 with DI water to reach the $\mathrm{pH}$ of the fresh cement paste used in this work, which was measured as 12.71 . The CNC pore solution suspensions were prepared with 7 different CNC concentrations as shown in the last column in Table 2 . These suspensions then can be regarded as the mixtures of CNCs and pore solution in the fresh cement pastes with cement taken out and the results can be directly related and compared with the experimental results of the cement pastes.

Table. 3. The 1 hour ionic composition in pore solution reported by Rajabipour et al. [28]

\begin{tabular}{|c|c|c|}
\hline ions & Conc. (mol/L) & Charge (mol/L) \\
\hline $\mathrm{K}^{+}$ & 0.43 & \multirow{2}{*}{0.57} \\
\hline $\mathrm{Na}^{+}$ & 0.10 & \\
\hline $\mathrm{Ca}^{2+}$ & 0.02 & \multirow{2}{*}{0.57} \\
\hline $\mathrm{OH}^{-}$ & 0.17 & \\
\hline$\left(\mathrm{SO}_{4}\right)^{2-}$ & 0.20 & \\
\hline
\end{tabular}

3.2. Ball-on-three-ball flexural test

The characterization of the flexural strength of the cement pastes was carried out with a ball-on-three-ball (B3B) flexural test. This technique has been described in a previous work [5]. In this testing set-up, the load is given by one ball pressuring downward at the center of a disc sample. Three ball supports are located beneath the sample in the corners of an equilateral triangle. There are two advantages of the B3B tests over traditional flexural tests performed on beam specimens: (1) the disk specimens for the B3B test can be easily obtained in large quantities from sectioning a cylinder; (2) the B3B test is more sensitive to defects in all the in-plane directions of the specimen [29].

The distribution of the CNCs within the cement paste matrix is related with both microstructural and mechanical 
properties. This section discusses the percolation threshold or critical concentration of CNCs using a combination of theoretical and experimental approaches relating the rheological properties to mechanical performance.

\subsubsection{Percolation threshold in the inert environment}

Garboczi et al. [15] used percolation theory to develop an expression to calculate percolation on only the

147 geometries of the inclusions in the matrix, regardless of their physical and chemical properties. The percolation threshold (in volume fraction) is dependent on the aspect ratio of the CNC (x) and can be determined by the following Pade-type formula:

$$
p_{c}=\frac{s x+x^{2}}{h+f x+g x^{3 / 2}+c x^{2}+d x^{3}}(\mathbf{1})
$$

151 where the coefficients are given as: $h$ 7.742, $f$ 14.61, $g$ 12.33, $c 1.763, d 1.658$, and $s$ 9.875. This model can be 152 employed to estimate the critical concentration of the CNCs in an inert environment such as deionized (DI) water 153 without influential factors like electrostatic force. The aspect ratio of CNC can vary within a wide range depending 154 on the source material and the processing technique. Goodsell et al. [3] suggested that the aspect ratio of CNC is 155 approximately 5 70. Araki et al. [30] reported an aspect ratio of 50 for the wood CNC. A TEM image by Moon et al. 156 [1] shows that a majority of CNCs extracted from wood have lengths of approximately $200 \mathrm{~nm}$. With a consensus in 157 literature that the width of $\mathrm{CNC}$ is $3 \sim 5 \mathrm{~nm}[1,2]$, the aspect ratio was adopted as 50 for calculation in this work.

158 Rheological experiments were conducted as described in Section 3.1 to investigate the percolation threshold of 159 CNCs in DI water, cement pore solution, and fresh cement pastes. Figure 2 shows the shear stress and the shear strain rate for CNC-DI water suspensions with concentrations ranging from 0 to $3.43 \mathrm{vol}$. \%. The shear stress

161 increases at all ranges of the shear strain rate with increasing CNC concentrations. This is because at low 162 concentrations, the CNCs are mostly in the form of free particles in the water and therefore have high mobility. With 163 increasing $\mathrm{CNC}$ concentrations there is an increased interaction between $\mathrm{CNCs}$ forming agglomerates or network 164 structures, which are to be broken or aligned during the rheological measurements and result in higher shear stress. 
Fig. 3. The relationship between the parameter $n$ and CNC concentration in DI water

Fig. 2. The shear stress and shear strain rate of CNC aqueous suspensions at different concentrations

\section{The shear stress to shear strain rate relationship of the CNC aqueous suspensions shows a shear thinning} behavior, especially for the higher CNC concentration suspensions. The behavior is typically described in the Herschel-Bulkley model, which gives the relationship between the shear stress and shear strain rate as:

$$
\tau=\tau_{0}+K \gamma^{n}(2)
$$

where $\tau$ is the shear stress, $\tau_{0}$ the yield stress, $\gamma$ the shear strain rate, $K$ the consistency, and $n$ the flow index [31, 32]. The flow index $n$ is directly related with the shear thinning or thickening behavior which happens for the nonNewtonian fluid: If $n>1$, the fluid is shear thickening; while $n<1$ the fluid is shear thinning [33] which is also referred as the pseudoplastic behavior [34]. All the shear stress to shear strain rate curves in Fig. 2 are fitted with the Herschel-Bulkley equation and the factor $n$ is plotted with the CNC concentrations in Fig. 3. The first data point with $0 \%$ of $\mathrm{CNC}$ is pure water, which is a typical Newtonian fluid and $n$ is 1 .

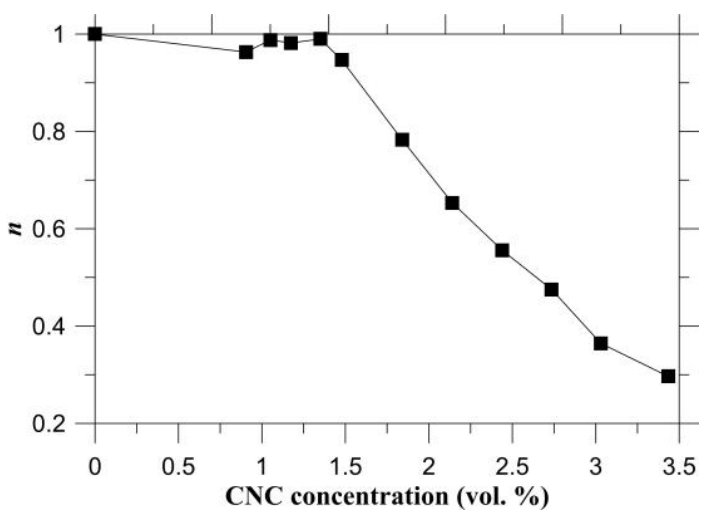

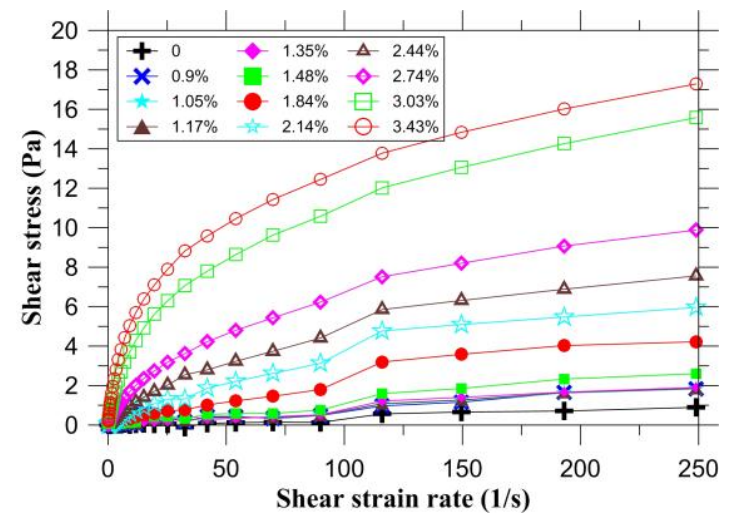


The relationship between the flow index $n$ and CNC concentration shows that $n$ is kept at a plateau with $n=1$ until the $\mathrm{CNC}$ concentration reaches a volume of $1.35 \%$. At higher concentration $n$ decreases. The variation in $n$ appears to indicate that a threshold of around $1.35 \%$ exists between the Newtonian and non-Newtonian (pseudoplastic) behavior. The origin of shear thinning is commonly thought to occur as a result of alignment or ordering of the particles, such as the formation of lines of particle (strings) parallel to the flow direction [35, 36], the alignment in the flow direction [37], and the formation of particle layers [38]. This suggests that for CNC concentration above $1.35 \%$ there an alignment of the CNCs and with increasing $\mathrm{CNC}$ concentration, the extent of this alignment and shear thinning increases. For concentrations below $1.35 \%$, shear thinning is not evident and it suggests that the CNCs do not form a significant amount of agglomerates and they are in the form of mobile particles that are easier to align. As such the flow index $n$ appears to be strongly related with CNC concentration and might be an indicator of percolation. It is found that based on the rheological measurements on the CNC-DI water suspensions, the percolation threshold occurs at $1.35 \%$, which agrees very well with the theoretical value $1.38 \%$ calculated from the geometrical percolation theory assuming the $\mathrm{CNC}$ has the aspect ratio of 50 (Eqn 1).

\subsubsection{Percolation threshold in the charged environment}

When CNC suspensions are mixed with cement, the rheology may differ from that of the CNC-DI water suspensions, since the solvent is no longer pure water. In the fresh cement paste, ionic species such as $\mathrm{K}^{+}, \mathrm{Na}^{+}, \mathrm{Ca}^{2+}$, $200 \mathrm{OH}^{-}$, and $\mathrm{SO}^{4-}$ exist in the pore solution and they are likely to change the surface charges on CNCs. The surface 201 charges result in an influence on the potential barrier induced by the repulsive Coulomb force between CNCs, and thus affect the agglomeration as well as the percolation threshold. Calcium nitrate is introduced into the CNC suspensions with increasing concentrations to investigate how ions affect the interaction and agglomeration of CNCs.

204 Two different CNC suspensions are prepared with the concentrations of 1.23 vol. $\%$ and 2.44 vol. \%, in which, the 205 former concentration is slightly lower than the percolation threshold 1.35 vol. \% while the latter is above that. The reason to choose these two specific concentrations is to evaluate the ionic influence on the CNC suspensions rheological properties at both the percolated and non-percolated states. The experimental design is shown in Table 4. 
Table 4. Experimental design of surface charged CNC solutions

\begin{tabular}{|c|c|c|c|c|c|c|c|c|c|}
\hline & \multicolumn{4}{|c|}{ Group 1 (CNC conc. = 1.23 vol. \%) } & \multicolumn{3}{c|}{ Group 2 (CNC conc. = 2.44 vol. \%) } \\
\hline ID & 1 & 2 & 3 & 4 & 5 & 7 & 8 & 9 & 10 \\
\hline $\mathrm{Ca}\left(\mathrm{NO}_{3}\right)_{2} /$ water (wt.) & $0 \%$ & $1 \%$ & $2 \%$ & $4 \%$ & $8 \%$ & $0 \%$ & $2 \%$ & $4 \%$ & $8 \%$ \\
\hline
\end{tabular}

210

211

212

213

214

215

216

217

After adding calcium nitrate into the CNC suspensions, all suspensions gelled immediately and are no longer transparent as Fig. 4 shows, while the references (\#1 and \#7) remain relatively transparent. Suspensions \#8, \#9 and \#10 seem extremely viscous as they tend to stick on the wall of the containers after mixing.

The rheological results for the suspensions are shown in Fig. 5. It is observed that: (1) the yield stresses are increased with the calcium nitrate concentration for both groups (Fig. 5 (a) and (b)). For the pure CNC suspensions, the yield stress is nearly 0 , while with calcium nitrate, Group 1 (Fig. 5(a)) gives a maximum yield stress of $4.5 \mathrm{~Pa}$ and Group 2 (Fig. 5(b)) shows a maximum of $25 \mathrm{~Pa}$. (2) The viscosities are also increased with the addition of calcium nitrate. The ionic concentration may alter the CNC surface charge and therefore change the rheological behavior, i.e., yield stress and viscosity, by making the CNCs adhere to each other and form more and/or larger agglomerates. The viscosities at 140 1/s and yield stresses of these suspensions are plotted in Fig. 6. It is observed that by increasing the calcium nitrate concentration, both viscosity and yield stress increase. For Group 2, which is above the percolation threshold, the increases are much greater than Group 1. It is found that the ions facilitate the CNC agglomeration, since the yield stress and viscosity are significantly increased from the CNC-DI water suspensions.

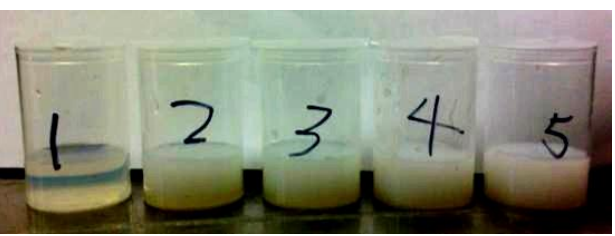

(a)

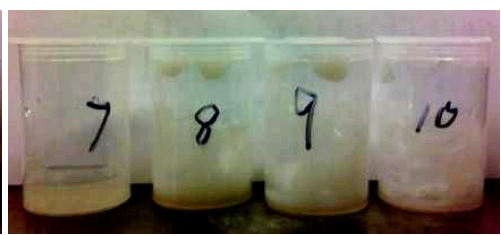

(b)

Fig. 4. Images of all CNC-Ca( $\left.\mathrm{NO}_{3}\right)_{2}$ suspension (a) Group 1 and (b) Group 2 


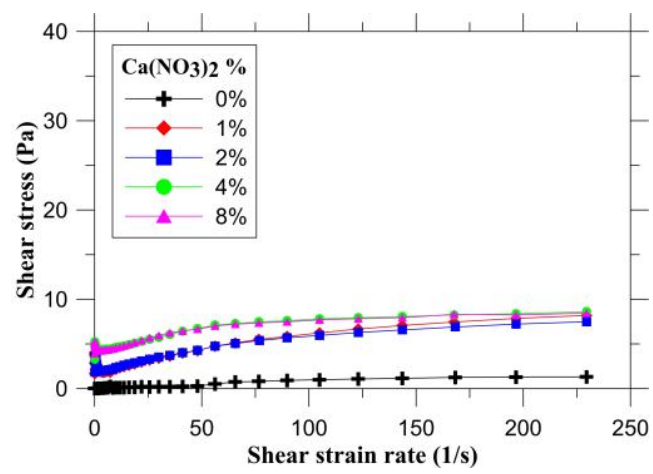

(a)

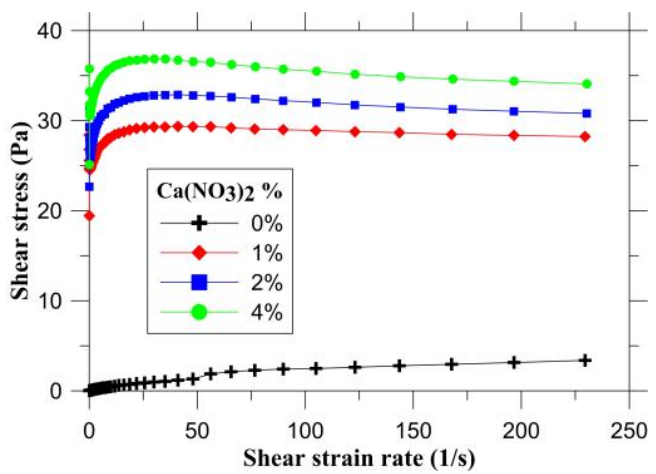

(b)

Fig. 5. Shear stress to shear strain rate relationships of the $\mathrm{CNC}-\mathrm{Ca}\left(\mathrm{NO}_{3}\right)_{2}$ aqueous suspensions with $\mathrm{CNC}$ concentration of (a) 1.23 vol. \%; (b) 2.44 vol. \%

The influence of the multiple ionic species on CNCs rheological performance is investigated according to the experimental design described in Section 3.1 to simulate the cement pore solution environment. The relationships between the shear stress and shear strain rate of the $7 \mathrm{CNC}$-pore solution suspensions are shown in Fig. 7. The viscosities at 140 1/s and the yield stress are plotted with the CNC concentration for both the CNC-DI water suspension and CNC-pore solution suspension (data from Fig. 2 and Fig. 7) in Fig. 8. It can be observed that with the same CNC concentrations, the pore solution suspension has much higher viscosities than the DI-water suspension. The yield stress for the CNC-pore solution suspensions is increased from nearly 0 to about $18.5 \mathrm{~Pa}$, while only an insignificant increase is observed for the CNC-DI water suspensions.

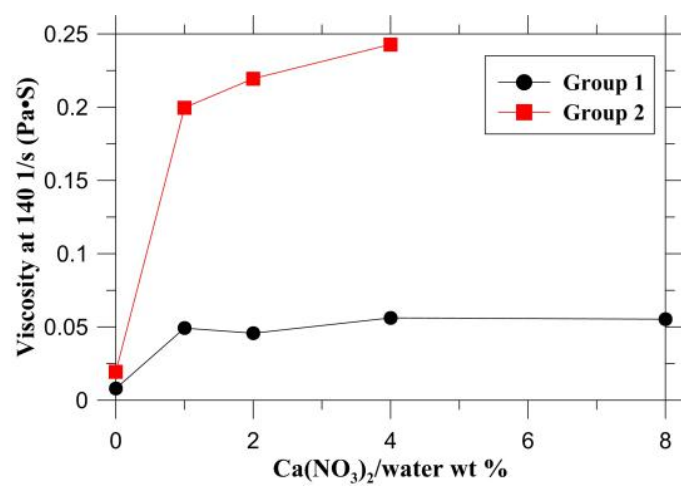

(a)

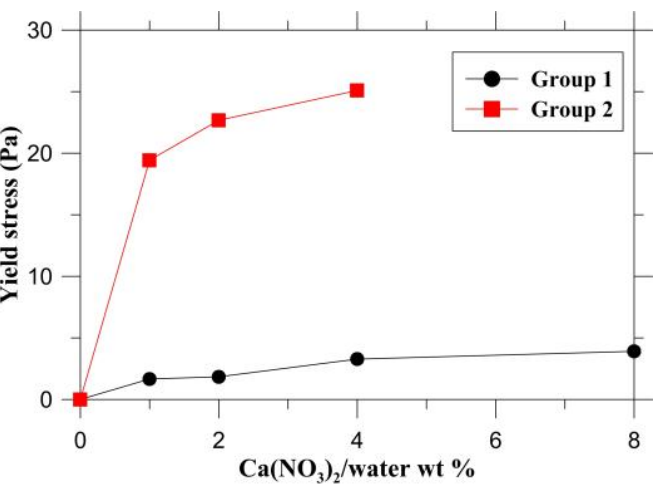

(b)

Fig. 6. (a) The viscosities at 140 1/s and (b) yield stress of Group 1 and Group 2 


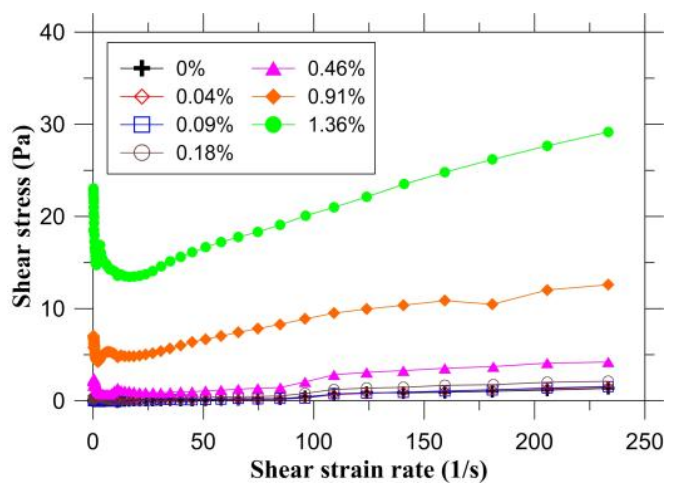

Fig. 7. Shear stress to shear strain rate relationships of the of pore solution with CNCs

It is observed from Fig. 7 that the shear stress to shear strain rate curves of the CNC-pore solution suspensions cannot be simply fitted with the Herschel-Bulkley function since a decrease in shear stress is found at the low shear rates and a minimum stress was achieved at the shear rate of about $20 \sim 251 / \mathrm{s}$. This is quite different from what was found for the solutions with only calcium nitrate (Fig. 5). Only the data above $251 / \mathrm{s}$ are fitted with the HerschelBulkley function and the flow index $n$ is plotted with the CNC concentration in Fig. 9. The result shows that above 0.46 vol. \% for the pseudoplastic behavior.

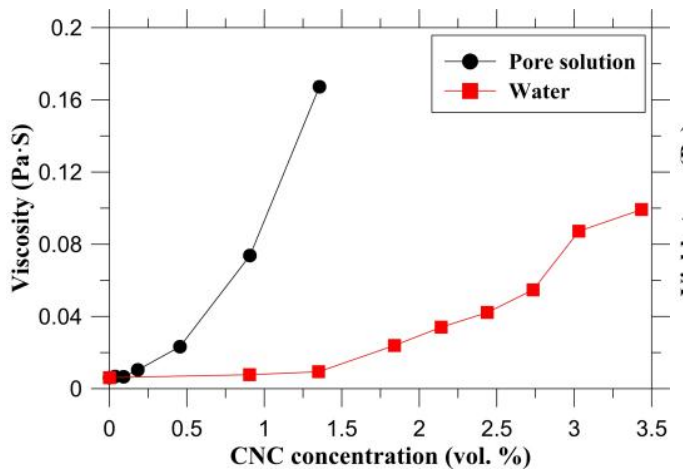

(a)

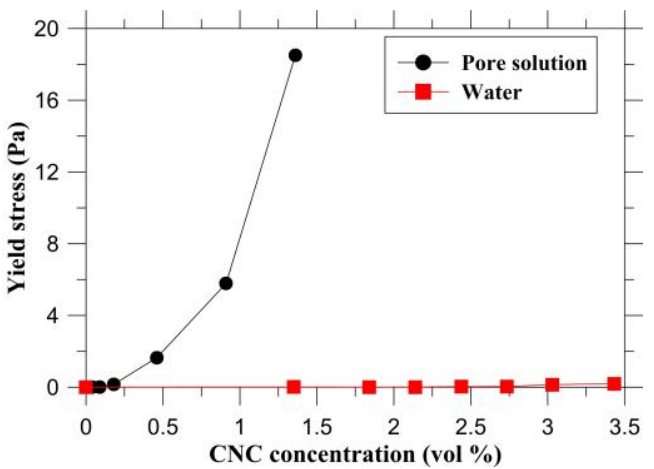

(b)

Fig. 8. (a) The viscosity at the strain rate of approximately $140 \mathrm{1} / \mathrm{s}$ and (b) the yield stress for the DI water and the 


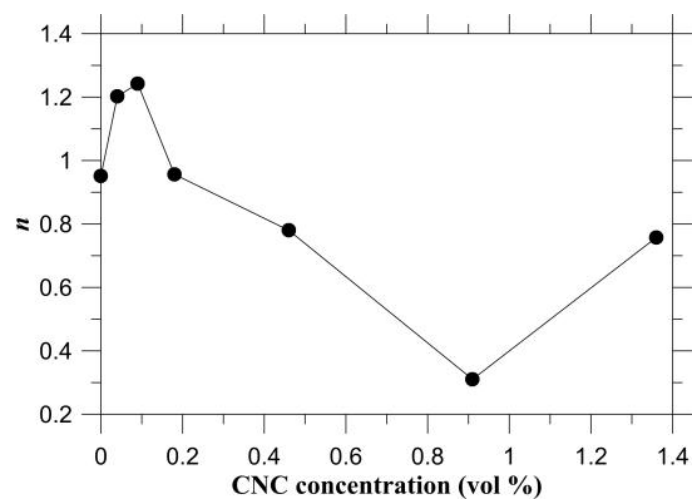

Fig. 9. The relationship between the parameter $n$ and $\mathrm{CNC}$ concentration in pore solution

These differences between the charged system and the DI water suspensions indicate that the surface charges result in agglomeration that yields to an increase both viscosity and yield stress of the mixture. From Table 2, the concentration 0.18 vol. \% of $\mathrm{CNC} /$ suspension corresponds to the $\mathrm{CNC} /$ cement concentration of 0.2 vol. \%, where the maximum strength was obtained [5]. For concentrations above $0.2 \mathrm{vol} \% \%$, the strength decreases due to a

271 considerable amount of $\mathrm{CNCs}$ agglomerates. The critical concentrations for CNCs agglomeration (percolation

272 threshold) are listed in Table 5. The rheological measurements show that the percolation threshold occurs at 273 approximately 1.35 vol. \% which agrees very well with the theoretical value 1.38 vol. \% calculated from the 274 geometrical percolation theory. Rheological studies of CNCs in calcium nitrate and simulated pore solutions show 275 that surface charges induce the agglomeration and percolation which is consistent with the concentration that results 276 in maximum flexural strength of paste [5]. The surface charges from pore solution decrease the percolation threshold 277 from about 1.35 vol. \% to around 0.18 0.46 vol. \%, above which concentration the agglomerates prevail. 
Table 5. Critical CNC concentrations obtained from different sources

\begin{tabular}{|c|c|c|c|c|}
\hline \multirow{2}{*}{ Matrix } & Data source & The meaning of "critical" & $\begin{array}{c}\text { CNC/cement } \\
(\mathrm{vol})\end{array}$ & $\begin{array}{c}\text { CNC/suspension } \\
\text { (vol) }\end{array}$ \\
\hline \multirow{3}{*}{ Inert } & Theoretical model & Percolation threshold & $/$ & $1.38 \%$ \\
\cline { 2 - 5 } & $n$ of DI Water suspension & Threshold for shear thinning & $/$ & $1.35 \%$ \\
\hline \multirow{3}{*}{ Charged } & $n$ of Pore solution suspension & Threshold for shear thinning & $/$ & $0.18 \% \sim 0.46 \%$ \\
\cline { 2 - 5 } & Yield stress of fresh cement & Agglomeration starts & \multirow{2}{*}{$0.2 \% \sim 0.5 \%$} & $0.18 \% \sim 0.46 \%$ \\
\cline { 2 - 5 } & Strength of hardened cement & developing & & $0.18 \%$ \\
\hline
\end{tabular}

\subsection{Dispersion of CNC}

The agglomeration of particles in cement composites limits the mechanical performance for the macro-level fibers [39] and carbon nanotubes [40]. This work used sonication to disperse the CNC agglomerates. Three different sonication durations $(0,5$, and $30 \mathrm{~min})$ were used on CNC-DI water suspensions as shown in Fig. 10. Different degrees of transparency were observed at the completion of sonication. This fact indicates that the longer sonication duration reduces the agglomerates.

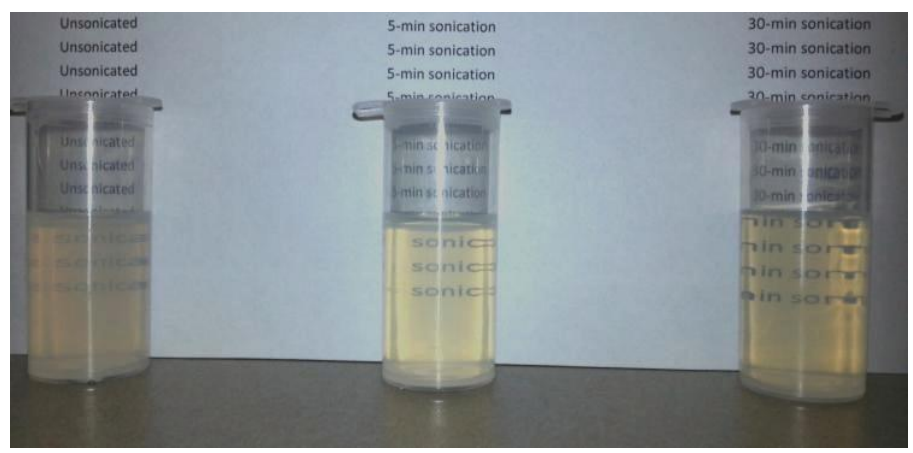

Fig. 10. Transparency of the CNC suspension is increasing with longer sonication

To quantitatively evaluate the degree of dispersion with sonication, rheological measurements are taken for 1.35 
overall shear stress decreases with increasing sonication time, which is indicative of the dispersion of the CNCs agglomerates, since the separated CNCs are relatively free and mobile compared with the ones in agglomerates.

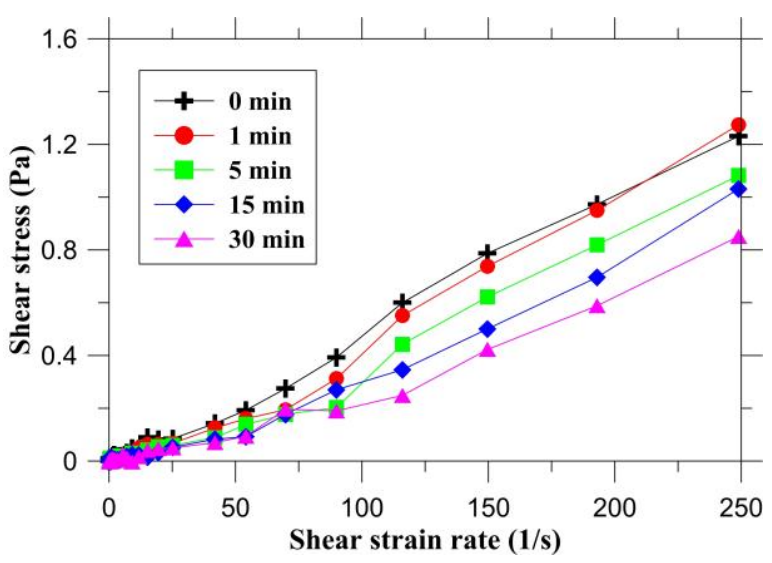

Fig. 11. The shear stress to shear strain rate relationships after sonication with different durations

A polycarboxylate-based WRA (brand name ADVA 140) was added in the suspensions to help facilitate the CNCs dispersion. Two different suspensions were prepared with different WRA/CNC mass ratios: 0.5 and 1 . The cement pastes with the sonicated CNCs with different amount of WRA were tested with a B3B flexural test to evaluate the dispersion effects of the WRA and their influence on the flexural strength.

\subsection{Flexural strength}

Figure 12 shows the flexural strengths of the cement pastes with the raw CNCs at different ages. The results show a maximum strength improvement by $20 \sim 30 \%$ at a concentration of approximately 0.2 vol. $\%$, which is consistent with the result reported in a previous work [5]. Figure 13 shows the flexural strengths with different sonication durations of 30 mintues (Fig. 13(a)) and 2 hours (Fig. 13(b)). The cement pastes with sonicated CNCs show higher strengths ( 50\% improvement) than the pastes with raw freeze dried CNCs for all ages and concentrations. More important, with high concentration of $\mathrm{CNC}$, i.e., $1.0 \mathrm{vol} . \%$ and 1.5 vol. $\%$, the strength keeps

311 increasing after the 0.2 vol. \% concentration. This implies that the CNC agglomerates are significantly reduced by 312 sonication that they are no longer in the cement paste acting as stress concentrators when a loading is applied. Figure 31314 summarized the 28-day flexural strengths of cement pastes with raw and ultra freeze dried and sonciated CNCs. 314 After soncation, the strengths are improved, especially at the high concentrations $(0.5 \%$ to $1.5 \%)$. 


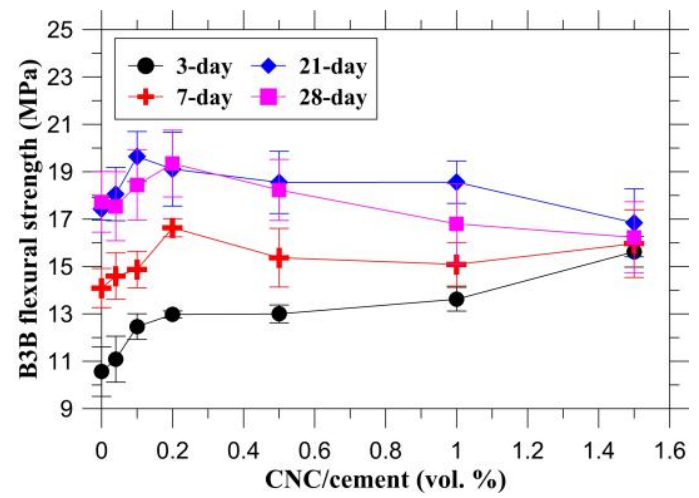

Fig. 12. B3B flexural strengths for the cement pastes with raw CNCs

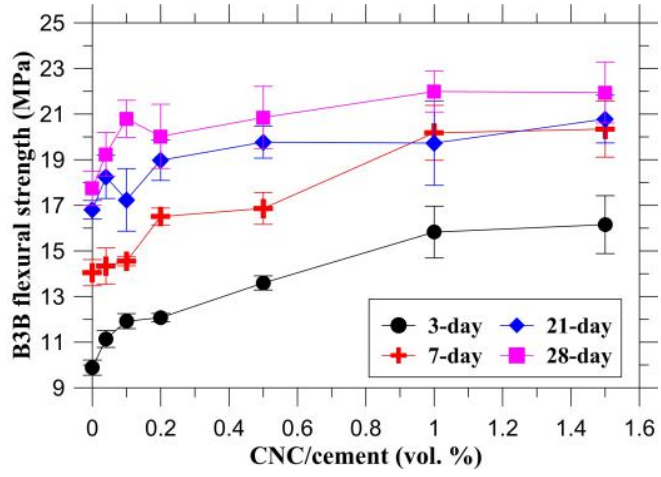

(a)

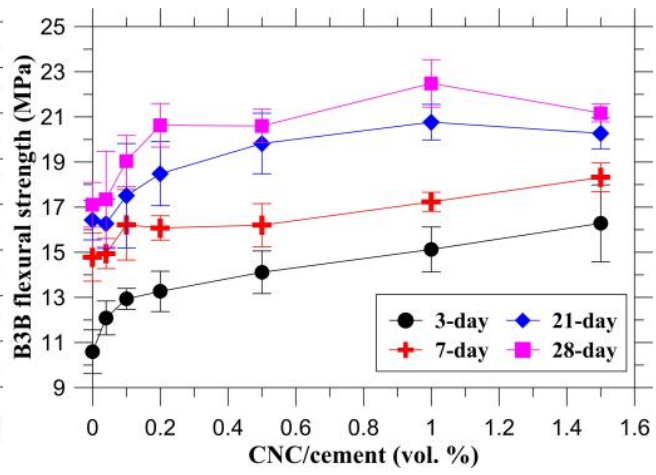

(b)

Fig. 13. B3B flexural strengths for the cement pastes with CNC sonicated (a) 30 minutes (b) 2 hours

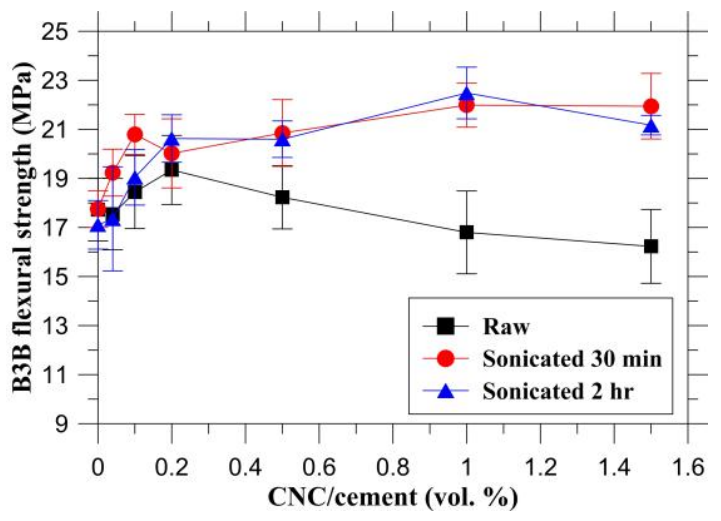

Fig. 14. 28-day B3B flexural strengths for the cement pastes with raw and sonicated CNCs 

without WRA.

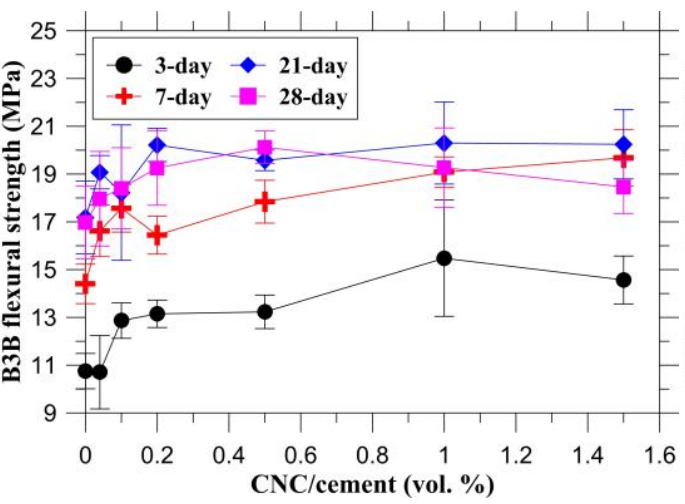

(a)

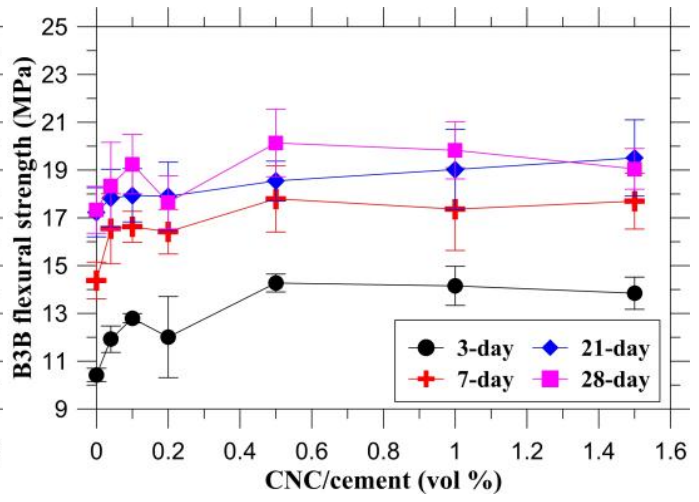

(b)

Fig. 15. B3B flexural strengths of the cement pastes with the sonicated CNC-WRA suspensions, WRA/CNC ratio of (a) 0.5 ; (b) 1 .

Figure 16 shows the relationship between the B3B flexural strengths and the DOH (degree of hydration) obtained from the isothermal calorimetry at the ages of 3 and 7 days. The DOH was estimated as the ratio Q/ $\mathrm{Q}_{\infty}$, where $\mathrm{Q}$ represents the cumulative heat released at a certain age and $\mathrm{Q}_{\infty}$ the theoretical amount of cumulative heat assumed the cement is completely hydrated [5]. $\mathrm{Q}_{\infty}$ is obtained by multiplying the theoretical value of each hydration component with the proportion of each component [41]. As expected, Fig. 16 shows an increase in strength with increased DOH. At the high concentration region (CNC addition of $1 \%$ and $1.5 \%$, highlighted with the dashed circles), the strength is greater with sonicated CNCs. This result presumes that sonication breaks the agglomerations and removes the defects acting as the stress concentrations. 


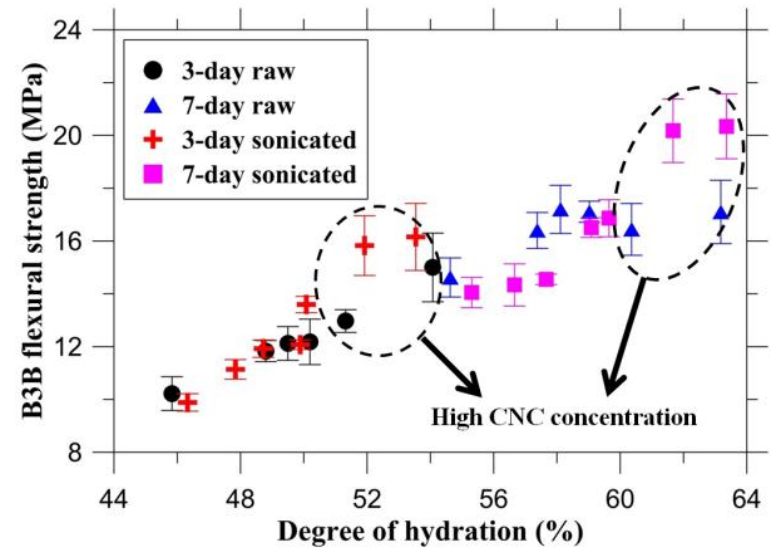

344 Fig. 16. The relationship between flexural strengths and DOH of cement pastes with raw and sonicated CNCs at the

age of 3 and 7 days

4. Conclusions

This paper attempts to relate the agglomeration of cellulose nanocrystals $(\mathrm{CNCs})$ in cement to the strengths of the hardened cement pastes. We examine whether ultrasonication can be used to reduce agglomerates and improve the mechanical performance. The concentration of CNC that causes agglomeration was examined. For CNCs in DI water a critical concentration of 1.35 vol. \% was found with rheological measurements, which agrees with the theoretical value 1.38 vol. \% calculated from the percolation model. When the CNCs were placed in a simulated cement pore solution, the critical concentration was reduced to approximately 0.18 vol. \%. Above this critical concentration, the agglomeration begins to occur and corresponds with the strength decrease of cement pastes. After ultrasonication, the dispersion of the CNCs improves the strength of the cement pastes by up to 50\%. The 50\% improvement is greater than the previously found improvement of $20 \sim 30 \%$ with the raw CNCs and indicate the dispersion of CNCs is key to improving the flexural strength of cement pastes with high concentration of CNCs.

\section{Acknowledgements}

This work was supported by the National Science Foundation through Awards no. CMMI \#1131596. The authors are grateful for the USFS Forest Products Laboratory for providing the CNC materials. We also thank Carlos Martinez for allowing the use of the rheometer. 


\section{References}

[1] Moon RJ, Martini A, Nairn J, Simonsen J, Youngblood J. Cellulose nanomaterials review: structure, properties and nanocomposites. Chem Soc Rev. 2011;40(7):3941-94.

[2] Habibi Y, Lucia LA, Rojas OJ. Cellulose nanocrystals: chemistry, self-assembly, and applications. Chem Rev. 2010;110(6):3479-500.

[3] Goodsell JE, Moon RJ, Huizar A, Pipes RB. A strategy for prediction of the elastic properties of epoxycellulose nanocrystal-reinforced fiber networks. Nord Pulp Pap Res J. 2014;29(1):85-94.

[4] Habibi Y, Dufresne A. Highly filled bionanocomposites from functionalized polysaccharide nanocrystals. Biomacromolecules. 2008;9(7):1974-80.

[5] Cao Y, Zavattieri PD, Youngblood J, Moon RJ, Weiss J. The influence of cellulose nanocrystal additions on the performance of cement paste. Cem Concr Compos. 2015;56:73-83.

[6] Broadbent S, Hammersley J. Percolation processes I. crystals and mazes. Proceedings of the Cambridge Philosophical Society1957.

[7] Kota AK, Cipriano BH, Duesterberg MK, Gershon AL, Powell D, Raghavan SR, et al. Electrical and rheological percolation in polystyrene/MWCNT nanocomposites. Macromolecules. 2007;40:7400-6.

[8] Schueler R, Petermann J, Schulte K, Wentzel H-P. Agglomeration and Electrical Percolation Behavior of Carbon Black Dispersed in Epoxy Resin. J Appl Polym Sci. 1997;63(13):1741-6.

[9] TARI Ö, PEKCAN Ö. A percolation approach for investigating the sol-gel phase transition of kcarrageenan: a steady-state fluorescence study. J Bioact Compat Polym. 2004;19:491-509.

[10] Bentz DP, Garboczi EJ. Percolation of phases in a three-dimensional cement paste microstructural model. Cem Concr Res. 1991;21(2):325-44.

[11] Scrivener KL, Nemati KM. The percolation of pore space in the cement paste/aggregate interfacial zone of concrete. Cem Concr Res. 1996;26(1):35-40.

[12] Xie P, Gu P, Beaudoin JJ. Electrical Percolation Phenomena in Cement Composites Containing Conductive Fibers. J Mater Sci. 1996;31:4093-7.

[13] Stauffer D, Aharony A. Introduction to Percolation Theory: Taylor \& Francis; 2003.

[14] Sandler JKW, Kirk JE, Kinloch IA, Shaffer MSP, Windle AH. Ultra-low electrical percolation threshold in carbon-nanotube-epoxy composites. Polymer. 2003;44:5893-9.

[15] Garboczi E, Snyder K, Douglas J. Geometrical percolation threshold of overlapping ellipsoids. Phys Rev E. 1995;52(1):819-28.

[16] Tian W, Yang R. Effect of interface scattering on phonon thermal conductivity percolation in random nanowire composites. Appl Phys Lett. 2007;90:2631051-3.

[17] Hsu WY, Holtje WG, Barkley JR. Percolation phenomena in polymer/carbon composites. J Mater Sci Lett. 1988;7(5):459-62.

[18] Lin C, Wang H, Yang W. Variable percolation threshold of composites with fiber fillers under compression. J Appl Phys. 2010;108:0135091-5.

[19] Sieradzki K, Bailey K, Alford TL. Agglomeration and percolation conductivity. Appl Phys Lett. 2001;79(21):3401-3.

[20] Schueler R, Petermann J, Schulte K, Wentzel H-P. Percolation in carbon black filled epoxy resin. Macromol Symp. 1996;104:261-8.

[21] Alig I, Skipa T, Lellinger D, Bierdel M, Meyer H. Dynamic percolation of carbon nanotube agglomerates in a polymer matrix: comparison of different model approaches. Phys Stat Sol (b). 2008;245(10):2264-7.

[22] Pegel S, Potschke P, Petzold G, Alig I, Dudkin SM, Lellinger D. Dispersion, agglomeration, and network formation of multiwalled carbon nanotubes in polycarbonate melts. Polymer. 2008;49:974-84. 
[23] Martin CA, Sandler JKW, Shaffer MSP, Schwarz MK, Bauhofer W, Schulte K, et al. Formation of percolating networks in multi-wall carbon-nanotube-epoxy composites. Compos Sci Technol. 2004;64:2309-16.

[24] Vaisman L, Wagner HD, Marom G. The role of surfactants in dispersion of carbon nanotubes. Adv Colloid Interface Sci. 2006:37-46.

[25] Konsta-Gdoutos M, Metaxa Z, Shah S. Multi-scale mechanical and fracture characteristics and earlyage strain capacity of high performance carbon nanotube/cement nanocomposites. Cem Concr Compos. 2010;32(2):110-5.

[26] Konsta-Gdoutos MS, Metaxa ZS, Shah SP. Highly dispersed carbon nanotube reinforced cement based materials. Cem Concr Res. 2010;40(7):1052-9.

[27] Hielscher T. Ultrasonic production of nano-size dispersions and emulsions. Proceedings of 1st Workshop on NanoTechnology Transfer in Europe. Paris, France: TIMA Editions; 2005.

[28] Rajabipour F, Sant G, Weiss J. Interactions between shrinkage reducing admixtures (SRA) and cement paste's pore solution. Cem Concr Res. 2008;38(5):606-15.

[29] Börger A, Supancic P, Danzer R. The ball on three balls test for strength testing of brittle discs: stress distribution in the disc. J Eur Ceram Soc. 2002;22(9):1425-36.

[30] Araki J, Wada M, Kuga S, Okano T. Flow properties of microcrystalline cellulose suspension prepared by acid treatment of native cellulose. Colloids Surf A. 1998;142:75-82.

[31] Herschel VWH, Bulkley R. Konsistenzmessungen von gummi-benzollösungen. Kolloid Z. 1926;39:291-300.

[32] Larson RG. The structure and rheology of complex fluids. New York: Oxford University Press, Inc.; 1999.

[33] Burgos GR, Alexandrou AN, Entov V. On the determination of yield surfaces in Herschel-Bulkley fluids. J Rheol. 1999;43(3):463-83.

[34] Cross MM. Rheology of non-Newtonian fluids: a new flow equation for pseudoplastic systems. J Colloid Sci. 1965;20:417-37.

[35] Visscher PB, Heyes DM. Growth of hexagonal string phases in sheared colloid simulation. J Chem Phys. 1994;101(7):6096-100.

[36] Phung TN, Brady JF, Bossis G. Stokesian dynamics simulation of brownian suspensions. J Fluid Mech. 1996;313:181-207.

[37] Forster S, Konrad M, Lindner P. Shear thinning and orientational ordering of wormlike micelles. Phys Rev Lett. 2005;97:0178031-4.

[38] Brader JM. Nonlinear rheology of colloidal dispersions. J Phys Condens Matter. 2010;22:1-37.

[39] Xu F, Zhou M, Chen J, Ruan S. Mechanical performance evaluation of polyester fiber and SBR latex compound-modified cement concrete road overlay material. Constr Build Mater. 2014;63:142-9.

[40] Andrews R, Weisenberger MC, Qian D, Meier MS, Cassity K, Yeary PE. Carbon nanotubes, multiwalled. In: Lukehart CM, Scott RA, editors. Nanomaterials: Inorganic and Bioinorganic Perspectives. United Kingdom: John Wiley \& Sons Ltd; 2008.

[41] Barnes P, Bensted J. Structure and performance of cements. New York: Spon Press, Taylor \& Francis Group; 2002. 